OPEN ACCESS

Edited by:

Qiang Wang,

Chinese Academy of Sciences, China

Reviewed by:

Jianhua Fan,

East China University of Science and Technology, China

Xiaowei Li,

Chalmers University of Technology,

Sweden

*Correspondence:

Zhen Gao

gaozhen@njtech.edu.cn

Xiao-Jun J

xiaojunji@njtech.edu.cn

Specialty section:

This article was submitted to Microbiotechnology, Ecotoxicology

and Bioremediation,

a section of the journal

Frontiers in Microbiology

Received: 31 July 2018

Accepted: 27 August 2018

Published: 20 September 2018

Citation:

Ji R-Y, Ding Y, Shi T-Q, Lin L,

Huang H, Gao Z and Ji X-J (2018)

Metabolic Engineering of Yeast for the Production

of 3-Hydroxypropionic Acid.

Front. Microbiol. 9:2185.

doi: 10.3389/fmicb.2018.02185

\section{Metabolic Engineering of Yeast for the Production of 3-Hydroxypropionic Acid}

\author{
Rong-Yu Ji', Ying Ding ${ }^{1}$, Tian-Qiong Shi ${ }^{1}$, Lu Lin ${ }^{2}$, He Huang ${ }^{2,3,4}$, Zhen Gao ${ }^{1 *}$ and \\ Xiao-Jun Ji ${ }^{1,4 *}$ \\ ${ }^{1}$ College of Biotechnology and Pharmaceutical Engineering, Nanjing Tech University, Nanjing, China, ${ }^{2}$ School \\ of Pharmaceutical Sciences, Nanjing Tech University, Nanjing, China, ${ }^{3}$ State Key Laboratory of Materials-Oriented Chemical \\ Engineering, Nanjing Tech University, Nanjing, China, ${ }^{4}$ Jiangsu National Synergetic Innovation Center for Advanced \\ Materials, Nanjing Tech University, Nanjing, China
}

The beta-hydroxy acid 3-hydroxypropionic acid (3-HP) is an attractive platform compound that can be used as a precursor for many commercially interesting compounds. In order to reduce the dependence on petroleum and follow sustainable development, 3-HP has been produced biologically from glucose or glycerol. It is reported that 3-HP synthesis pathways can be constructed in microbes such as Escherichia coli, Klebsiella pneumoniae and the yeast Saccharomyces cerevisiae. Among these host strains, yeast is prominent because of its strong acid tolerance which can simplify the fermentation process. Currently, the malonyl-CoA reductase pathway and the $\beta$-alanine pathway have been successfully constructed in yeast. This review presents the current developments in 3-HP production using yeast as an industrial host. By combining genome-scale engineering tools, malonyl-CoA biosensors and optimization of downstream fermentation, the production of 3-HP in yeast has the potential to reach or even exceed the yield of chemical production in the future.

Keywords: 3-Hydroxypropionic acid, metabolic engineering, yeast, malonyl-CoA reductase pathway, $\beta$-alanine pathway

\section{INTRODUCTION}

With the reduction of easily obtainable fossil fuels and sustainable development becoming the mainstream of the world, petroleum-based compounds are gradually being replaced by bio-based compounds to meet the increasing demands for bulk materials and fine chemicals alike (Nielsen and Keasling, 2016; Chen et al., 2018a). Increasingly sophisticated cell factories are being built to produce many renewable bio-based chemicals. Among them, 3-hydroxypropionic acid (3HP) ranked third in the top 12 value-added chemicals from biomass listed by the United States Department of Energy in 2004 (Werpy et al., 2004). As a hydroxy-functionalized carboxylic acid, 3-HP is a promising platform chemical, which can be transformed to a variety of worthy products such as malonic acid, acrylic acid, acrylic esters, 1,3-propanediol, and building blocks for biodegradable polymers (Liu et al., 2017). The two most important present applications of 3-HP are the production of acrylic acid and poly(3-hydroxypropionic acid) ( $\mathrm{P}[3-\mathrm{HP}])$. Acrylic acid is able to be obtained by dehydrating 3-HP, and acrylic acid-derived products are widely used in paints, paper, baby diapers, adhesives, textiles, specialty coatings, and superabsor. While $\mathrm{P}$ [3-HP] has good mechanical properties and can be hydrolyzed enzymatically, mainly used for making surgical products (Zhang et al., 2004; Pina et al., 2011). It has been pointed out 
that 3-HP has a wide market and great application value. It can be obtained by chemical synthesis and biosynthesis methods. Traditional chemical synthesis of 3-HP is well-established, but resource limitations, high price and the toxicity of the used raw materials do not meet the new sustainable development goals. Compared with chemical synthesis, biosynthesis methods have many advantages, including cheaper substrates, mild reaction conditions and simple operation, which are compatible with sustainable development, and the reduced amounts of byproducts help reduce the cost of production.

In recent years, the biosynthesis of 3-HP has attracted wide attention, which led to rapid new developments. First discovered as an intermediate metabolite of the 3-HP cycle in Chloroflexus aurantiacus, 3-HP was also found in Acidianus brierleyi, Acidianus ambivalens, and Sulfolobus metallicus (Holo, 1989; Strauss et al., 1992; Jiang et al., 2009).

Up till now, three known pathways for 3-HP production have been constructed in recombinant organisms, the glycerol pathway, the $\beta$-alanine pathway, and the malonyl-CoA reductase (MCR) pathway. The natural generation of 3-HP proceeds through the glycerol pathway (Lan et al., 2015). However, the intermediate metabolite 3-hydroxypropionaldehyde (3-HPA) is toxic to cells and requires an efficient aldehyde dehydrogenase to convert it to 3-HP. To prevent the accumulation of this toxic intermediate, current emphasis is on balancing the enzymes' expression (Jiang et al., 2009). The MCR pathway, which used the intermediate malonyl-CoA as the substrate, only involves one enzymatic reaction, mainly depending on MCR (Chen et al., 2014; Lian et al., 2018). However, the $\beta$-alanine pathway is considered to be the most economically attractive (Borodina et al., 2015).

Three common hosts for the biosynthesis of 3-HP from glucose and/or glycerol are Escherichia coli, Klebsiella pneumoniae, and the yeast Saccharomyces cerevisiae. Although $E$. coli is the most widely studied, there are many limitations slowing further research. In E. coli, the conversion of glycerol to 3 - $\mathrm{HP}$ requires to add additional coenzyme $\mathrm{B}_{12}$ to the culture medium, which is sensitive to oxygen and replaced by vitamin $\mathrm{B}_{12}$ because of the aerobic conditions required for 3-HP production (Roth et al., 1996; Daniel et al., 1998; Toraya, 2002; Rathnasingh et al., 2012; Kumar et al., 2013; Chen et al., 2014; Ko et al., 2014). Since E. coli cannot produce coenzyme $\mathrm{B}_{12}$, it requires exogenous addition, which increases the cost of industrial production significantly (Valdehuesa et al., 2013). K. pneumoniae is another alternative host, and it can synthesize coenzyme $\mathrm{B}_{12}$ naturally (Kumar et al., 2013). However, in addition to being a pathogenic bacterium, it is also a challenge for $K$. pneumoniae to balance electron transportation from $\mathrm{NADH}$ to $\mathrm{NAD}^{+}$with the oxygen demand and the decrease of coenzyme $B_{12}$ production under aerobic conditions (Kumar et al., 2013; Chen and Nielsen, 2016). Moreover, the $\mathrm{pH}$ decrease due to the production of 3-HP in E. coli and K. pneumoniae leads to an acidic intracellular $\mathrm{pH}$, which seriously affects cell growth, resulting in reduced $3-\mathrm{HP}$ tolerance. The toxicity of 3-HP requires the addition of a large amount of base titrant into the cultures when using these bacteria. By contrast, yeast has a strong intrinsic tolerance to low $\mathrm{pH}$, and recombinant strains based on yeast are not affected by 3-HP toxicity, significantly reducing the fermentation cost (Liu et al., 2017). Kildegaard et al. (2014) proposed that 3-HP could be converted to toxic 3-HPA by aldehyde dehydrogenases and subsequently detoxified through glutathione-dependent reactions in yeast. Due to its advantages of high tolerance to relatively low $\mathrm{pH}$, available whole-genome sequences of multiple strains, and easy molecular genetic manipulation, yeast is perhaps the most promising host strain for 3-HP production (Liu et al., 2017).

\section{ENGINEERING YEAST FOR EFFICIENT 3-HP PRODUCTION}

As shown in Figure 1, two efficient pathways for 3-HP production from glucose have been constructed in yeast the MCR pathway and the $\beta$-alanine pathway, and the main metabolic engineering strategies are summarized in Table 1.

\section{The Malonyl-CoA Reductase Pathway}

Recently, the MCR pathway has been successfully constructed in yeast. This pathway relies on the conversion of glucose into pyruvate by glycolysis. The pyruvate is used to generate acetaldehyde by PDC (pyruvate dehydrogenase complex), then acetate is formed by oxidation of $\mathrm{ADH}$ (aldehyde dehydrogenase) and further converted to acetyl-CoA by ACS (acetyl-CoA synthetase) (Tang et al., 2015). As the most important precursor of 3-HP production, malonyl-CoA is generated from acetyl-CoA by ACC1 (acetyl-CoA carboxylase), and the final product 3-HP is produced via a two-step reaction catalyzed by MCR. MCR is a bi-functional enzyme, consisting of two short-chain domains, the $\mathrm{N}$-terminal domain encoding an alcohol dehydrogenase and the C-terminal encoding an aldehyde dehydrogenase. The production of 3-HP was successfully increased by balancing these two enzyme domains (Hügler et al., 2002; Liu et al., 2013, 2016). The production of malonyl-CoA is strictly regulated in yeast, and its yield in the cytoplasm directly affects the 3-HP production. Therefore, it is meaningful to increase the malonylCoA concentration in the cytoplasm.

\section{Improving the Supply of the Precursor Acetyl-CoA}

Malonyl-CoA is an important substrate for the production of 3-HP by MCR. It is mainly produced from acetyl-CoA by ACC1, and known as a flux-controlling step (Tehlivets et al., 2007; Chen et al., 2018b). Therefore, the accumulation of acetyl-CoA in the cytoplasm has a significant impact on the synthesis of 3-HP. In S. cerevisiae, pyruvate in the cytoplasm can be converted to acetaldehyde through decarboxylation, thus further generate acetyl-CoA. And the acetaldehyde can further converted to ethanol by alcohol dehydrogenase, resulting in the so-called Crabtree effect (Hoek et al., 1998; Chen et al., 2013). Studies of acetyl-CoA metabolism have shown that it is quite complex in yeast, where it can be produced in the mitochondria, peroxisomes, and the cytosol (Pronk et al., 1996; Chen et al., 2012). In S. cerevisiae, acetyl-CoA cannot be transported to different compartments spontaneously, and instead utilizes a carnitine/acetyl-carnitine shuttle or the 


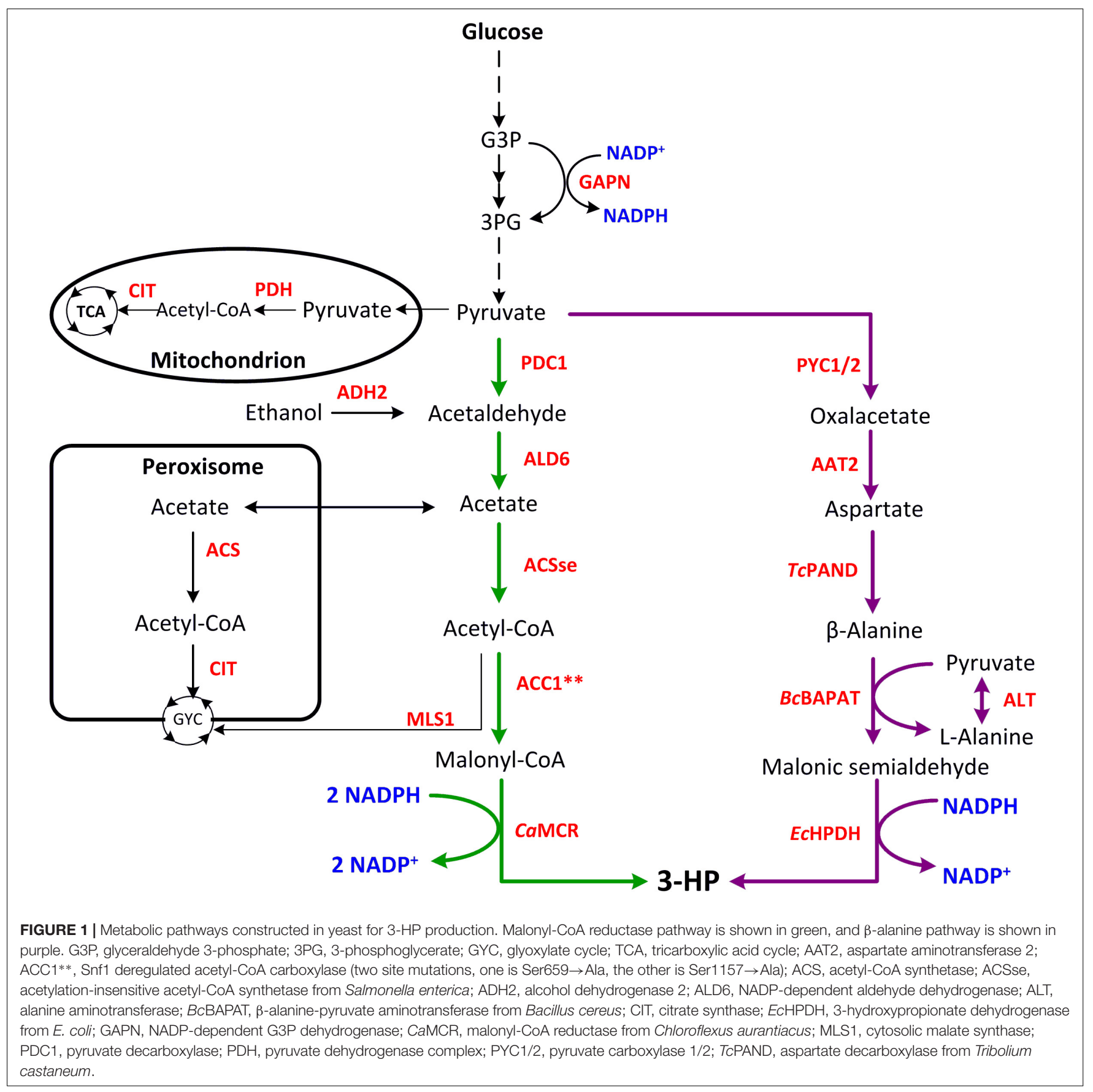

glyoxylate cycle (Chen et al., 2013). Therefore, many studies have focused on increasing the amount of acetyl-CoA in the cytoplasm.

Three strategies have been proposed to increase the accumulation of acetyl-CoA in the cytoplasm. The first strategy relies on pulling carbon into the cytosolic pool (Kocharin et al., 2012; Chen et al., 2013). Ethanol is the main metabolite of $S$. cerevisiae, but a by-product in the production of 3$\mathrm{HP}$. When the endogenous $\mathrm{ADH} 2$ (alcohol dehydrogenase) was co-expressed with ALD6 (NADP-dependent aldehyde dehydrogenase), ACC1 and a codon optimized ACS variant,
ACSse (an acetylation-insensitive acetyl-CoA synthetase from Salmonella enterica), the flux was successfully redirected from ethanol to acetyl-CoA in the cytosol, which improved 3-HP production to $210 \mathrm{mg} / \mathrm{L}$, up to twofold of the control strain (Chen et al., 2014). It is reported that intracellular acetyl-CoA levels could be increased to 2.19 fold by overexpression of ACS1 (acetyl-CoA synthetase 1) in S. cerevisiae, and improved to 5.02 fold by overexpression of ACS2 (acetyl-CoA synthetase 2) (Chen et al., 2013). ACC1 activity is repressed by Snf1 protein kinase at the protein level, as phosphorylation by Snf1 inactivates ACC1 (Woods et al., 1994). Shi et al. (2014) improved the activity 
TABLE 1 | Metabolic engineering strategies for 3-hydroxypropionate production in yeast.

\begin{tabular}{|c|c|c|}
\hline Metabolic engineering strategies & Beneficial effect for 3-HP production & Reference \\
\hline $\begin{array}{l}\text { Increasing the supply of malonyl-CoA: Overexpression the enzymes } \\
\text { (ALD6, ACSse, ADH2) for acetyl-CoA accumulation; Deleting the MLS1 } \\
\text { to block the consumption of acetyl-CoA; Overexpression the ACC1 } \\
\text { catalyzing acetyl-CoA to malonyl-CoA. Increasing the supply of } \\
\text { NADPH: Overexpression the GAPN catalyzing the formation of extra } \\
\text { NADPH. Establishing the MCR pathway: Overexpression the CaMCR. }\end{array}$ & 3-HP production was increased to $463 \mathrm{mg} / \mathrm{L}$ & Chen et al., 2014 \\
\hline $\begin{array}{l}\text { Generating the ACC1 mutant (ACC1Ser659Ala Ser1157Ala) through } \\
\text { mutating the potential phosphorylation sites to abolish the } \\
\text { post-translational regulation Increasing the ACC1 activity by } \\
\text { overexpression of the ACC1 mutant }\end{array}$ & $\begin{array}{l}\text { 3-HP production was up to } \sim 2.2 \text {-fold more than that of the } \\
\text { wild-type ACC1 }\end{array}$ & Shi et al., 2014 \\
\hline $\begin{array}{l}\text { Constructing the } \beta \text {-alanine pathway by overexpressing AAT2, PYC1, } \\
\text { PYC2, ALT, BcBAPAT, ECHPDH, and multiple copies of TCPAND }\end{array}$ & $\begin{array}{l}13.7 \mathrm{~g} / \mathrm{L} 3-\mathrm{HP} \text { was generated through the constructed } \\
\beta \text {-alanine pathway from glucose in fed-batch fermentation } \\
\text { at low } \mathrm{pH} \text {. }\end{array}$ & Borodina et al., 2015 \\
\hline $\begin{array}{l}\text { Increasing the supply of acetyl-CoA by overexpressing PDC1, ALD6, } \\
\text { and ACSse; Engineering the cofactor specificity of the GAPN to } \\
\text { increase the production of NADPH at the expense of NADH and thus } \\
\text { improve 3-HP production and reduce formation of glycerol as } \\
\text { by-product; Integrating multiple copies of CaMCR and ACC1 mutant } \\
\text { genes into the genome. }\end{array}$ & $\begin{array}{l}\text { 3-HP was produced at a titer } 7.37 \mathrm{~g} / \mathrm{L} \text { in a carbon-limited } \\
\text { fed-batch fermentation }\end{array}$ & Kildegaard et al., 2016 \\
\hline $\begin{array}{l}\text { Developing a malonyl-CoA biosensor based on the bacterial } \\
\text { transcription factor FapR to monitor and precisely control the } \\
\text { intracellular malonyl-CoA concentration }\end{array}$ & 3-HP titer was enhanced i by $120 \%$ & Li et al., 2015 \\
\hline $\begin{array}{l}\text { A hierarchical dynamic control strategy to control the expression level of } \\
\text { CaMCR depending on the intracellular malonyl-CoA concentration: The } \\
\text { upper level of control was to dynamically downregulate fatty acid } \\
\text { biosynthesis using HXT1 promoter. The lower level was based on the } \\
\text { malonyl-CoA biosensor. }\end{array}$ & 3-HP production was increased by 10 -fold & David et al., 2016 \\
\hline $\begin{array}{l}\text { Improving the availability of malonyl-CoA through down-regulating lipid } \\
\text { synthesis. Manipulating the phospholipid synthesis transcriptional } \\
\text { regulators including Ino2p, Ino4p, Opi1p, and a series of synthetic } \\
\text { Ino2p variants, combining with studying the inositol and choline effect. }\end{array}$ & 3-HP production was increased by 9-fold & Chen et al., 2017 \\
\hline $\begin{array}{l}\text { Identifying and characterizing promoters that depend on glucose } \\
\text { concentration for use as dynamic control elements. Identifying } 34 \\
\text { candidate promoters that strongly responded to glucose presence or } \\
\text { absence. A subset of promoters, pADH2, pICL1, and pHXT7, were } \\
\text { demonstrated as suitable for dynamic control of 3-HP production. }\end{array}$ & $\begin{array}{l}\text { Regulating the } 3-\mathrm{HP} \text { pathway by the ICL1 promoter } \\
\text { resulted in } 70 \% \text { improvement of } 3-\mathrm{HP} \text { titer in comparison to } \\
\text { PGK1 promoter. }\end{array}$ & Maury et al., 2018 \\
\hline
\end{tabular}
castaneum

of ACC1 in S. cerevisiae by abolishing the Snf1-dependent regulation through mutating the target sites of Snf1-mediated phosphorylation (Ser659 $\rightarrow$ Ala, and Ser1157 $\rightarrow$ Ala). Further overexpression of the mutated ACC1 ( $\mathrm{ACC}^{* *}$ ) increased the 3-HP titer to $\sim 279 \mathrm{mg} / \mathrm{L}$, increased more than 2.2 -fold in comparison with the wild-type.

The second strategy relies on reducing the consumption of acetyl-CoA in the cytoplasm by other accessory metabolic pathways. Acetyl-CoA is produced in four different compartments of the $S$. cerevisiae cells by different metabolic mechanisms. Pyruvate from the cytoplasm generates acetylCoA by PDH and then enters the tricarboxylic acid (TCA) cycle via CIT (citrate synthase). In the peroxisomes, acetyl-CoA is generated from acetate by ACS and then enters the glyoxylate cycle via CIT. Part of the cytoplasmic acetyl-CoA also enters the glyoxylate cycle via MLS (malate synthase) (Nielsen, 2014). To minimize the loss of acetyl-CoA in the cytoplasm, CIT2 and
MLS1, which encode the key enzymes of the glyoxylate cycle, were individually knocked out (Chen et al., 2012, 2013). Based on the over-expression of ACC1, ACSse, ADH2 and ALD6, the 3-HP titer could reach $249 \mathrm{mg} / \mathrm{L}$ when combined with deletion of CIT2, and reach $270 \mathrm{mg} / \mathrm{L}$ when combined with deletion of MLS1 (Chen et al., 2014).

The last strategy entails constructing new acetyl-CoA pathways in the cytoplasm. The pyruvate in the cytoplasm is partially converted into acetate by PDC (pyruvate decarboxylase), partly enters the mitochondria to produce acetyl-CoA via the $\mathrm{PDH}$. Although the $\mathrm{PDH}$ is only present in the mitochondria, if there is no MTS (mitochondrial targeting sequence) in the structural genes, $\mathrm{PDH}$ in the mitochondria will be redirected to the cytoplasm (cytoPDH), thus constructing a new pathway to generate acetyl-CoA directly from pyruvate. In one study, an MTS-free PDH structural genes from S. cerevisiae was used in combination with the PDH from Enterococcus faecalis to 
successfully increase the content of acetyl-CoA for the production of n-butanol (Lian et al., 2014).

\section{Improving the Supply of the Cofactor NADPH}

$\mathrm{NADPH}$ is another important precursor, functioned as the cofactor, for 3-HP production through the MCR pathway. In this pathway, 2 mol NADPH was needed to convert malonyl-CoA to 3-HP by MCR (Hügler et al., 2002; Valdehuesa et al., 2013; Lynch et al., 2016). It has been shown that the availability of NADPH for the production of 3-HP can be increased effectively by heterologous expression of a non-phosphorylating NADPdependent glyceraldehyde-3-phosphate dehydrogenase (GAPN) (Bro et al., 2006; Guo et al., 2011). Moreover, although the expression of GAPN can improve the content of NADPH, it has no effect on the production and biomass yield (Verho et al., 2003; Chen et al., 2014). When the expression of GAPN was coupled with improved precursor supply, the 3-HP titer was promoted to $463 \mathrm{mg} / \mathrm{L}$ (Chen et al., 2014).

\section{Precisely Control the Supply of Malonyl-CoA}

Malonyl-CoA is the direct precursor of 3-HP in the MCR pathway. It is strictly regulated in yeast and its concentration is maintained at a low level. Therefore, increasing the cytosolic malonyl-CoA content is extremely important for improving the production of the malonyl-CoA derived 3-HP.

To better manipulate malonyl-CoA levels, monitoring and enhancing the content of malonyl-CoA in real time is vital. In one approach, a malonyl-CoA sensor was developed based on the FapR transcription factor from Bacillus subtilis by Li et al. (2015). They designed the sensor using a codon-optimized bacterial transcription factor FapR and its corresponding operator fapO to sense intracellular malonyl-CoA levels in S. cerevisiae for the first time ( $\mathrm{Li}$ et al., 2015). They combined the biosensor with a genome-scale cDNA overexpression library, identifying two key genes that affect the levels of malonyl-CoA, TPI1 and PMP1. Overexpression of the key genes increased the malonylCoA concentration, further enhancing the 3-HP production by $120 \%$. This sensor provides a novel way to study the complex malonyl-CoA metabolism and to enhance the 3-HP production. Utilizing the malonyl-CoA sensor, David et al. (2016) established a hierarchical dynamic pathway control system on the genetic level for 3-HP production in S. cerevisiae. They integrated two different levels of dynamic control. The upper level of control was used to dynamically downregulate fatty acid biosynthesis using the HXT1 promoter to modifying the FAS1 (fatty acid synthase 1) gene expression characteristics. The lower level was based on the malonyl-CoA biosensor, which was used to link the expression levels of MCR to the intracellular malonyl-CoA concentration. This novel approach significantly improved the production of 3-HP 10-fold in yeast. Chen et al. (2017) successfully improved the availability of malonyl-CoA through down-regulating the lipid synthesis. They manipulated the transcriptional regulators for phospholipid synthesis, including Ino2p, Ino4p, Opilp, and a series of synthetic Ino2p variants, to control the malonylCoA levels and increase the synthesis of malonyl-CoA-derived products. The engineered strain in which multiple regulators were manipulated showed a 9-fold increase of the 3-HP titer. The study thus provides a novel strategy to regulate the intracellular malonyl-CoA availability and the 3-HP production. Maury et al. (2018) identified and characterized the yeast promoters that depend on glucose concentration, i.e., they are strongly responded to glucose presence or absence, and tried to use them as dynamic control elements for malonyl-CoA derived 3-HP production.

\section{The $\beta$-Alanine Pathway}

Another 3-HP synthesis pathway constructed in yeast is the $\beta$-alanine pathway (Figure 1). Researchers from Cargill Inc. evaluated three synthetic pathways for 3-HP production which utilize $\beta$-alanine as an intermediate metabolite (Liao et al., 2007; Gokarn et al., 2011). Two of these are completely unfeasible in thermodynamic terms, while the third pathway is relatively short and appeared feasible. However, due to the relatively complex synthesis of $\beta$-alanine by the organism itself, the efficiency of 3-HP synthesis remained very low. Nevertheless, many studies have optimized this pathway and improved the production of 3-HP in yeast (Burk and Osterhout, 2010). This way, glucose is converted into pyruvate through glycolysis, which in turn is used to generate $\beta$-alanine in three steps. The $\beta$-alanine is derived from aspartate by the action of PAND (aspartate decarboxylase), which is encoded by the pabD gene in bacteria but does not exist in yeast.

Jessen et al. (2015) proposed that the transformation of $\beta$-alanine to malonic semialdehyde relies on GABT $(\gamma$-aminobutyrate transaminase) and BAPAT ( $\beta$-alaninepyruvate aminotransferase). Subsequently, malonic semialdehyde is further dehydrogenated by HPDH (3hydroxypropionate dehydrogenase or 3-hydroxyisobutyrate dehydrogenase) to yield 3-HP (Borodina et al., 2015). In the whole pathway, the NADPH produced by glycolysis is later consumed by HPDH, and just 1 mol ATP is generated (Kumar et al., 2013). Therefore, to improve the 3-HP production through the $\beta$-alanine pathway must resort to the heterologous enzymes. The BAPAT activity has a large influence on the production of 3-HP. BcBAPAT ( $\beta$-alanine-pyruvate aminotransferase from Bacillus cereus) is considered to be the most active enzyme, comparing favorably with the BAPAT homologs from Pseudomonas putida and P. aeruginosa (Liao et al., 2007, 2010). EcHPDH (3-hydroxypropanoate dehydrogenase from E. coli) is another efficient enzyme for the conversion of $\beta$-alanine into 3-HP. Aspartate-1-decarboxylases from different sources were also tested to enable $\beta$-alanine biosynthesis from aspartate, among which TcPAND (aspartate-1-decarboxylase from Tribolium castaneum) is the most efficient (Lian et al., 2018). Overexpression of endogenous PYC (pyruvate carboxylase) and AAT2 (cytoplasmic aspartate aminotransferase) can be used to effectively channel the flux from pyruvate to aspartate. Overexpression of 5 genes, PYC1, PYC2, BcBAPAT, TcPAND, and $P p H I B A D H \quad$ (NADH-dependent 3-hydroxyisobutyrate dehydrogenase from $P$. putida) yielded $\sim 0.6 \mathrm{~g} / \mathrm{L}$ and $\sim 0.49 \mathrm{~g} / \mathrm{L}$ 3-HP from glucose and xylose, respectively (Kildegaard et al., 2015). A 3-HP high-producing strain can be obtained by overexpressing five genes, AAT2, BcBAPAT, EcHPDH, and PYC1/2, together with multiple copies of TcPAND, producing 
$12.2 \mathrm{~g} / \mathrm{L}$ and $13.7 \mathrm{~g} / \mathrm{L}$ of $3-\mathrm{HP}$ by deep fermentation and controlled fed-batch fermentation at $\mathrm{pH} 5.0$ with glucose as the substrate, respectively (Borodina et al., 2015).

\section{CONCLUSION AND PERSPECTIVES}

The biosynthesis of 3-HP has developed rapidly in recent years, and two synthetic pathways of 3-HP, the MCR and $\beta$-alanine pathways, have been established in yeast by rational metabolic engineering. Using the MCR pathway, the yield of 3 -HP could be increased up to $\sim 10 \mathrm{~g} / \mathrm{L}$ by increasing the supply of the precursors acetyl-CoA and NADPH, as well as optimizing the medium and culture conditions. Similarly, the yield of 3-HP in the $\beta$-alanine pathway reached $13.7 \mathrm{~g} / \mathrm{L}$ in the controlled fed-batch fermentations at $\mathrm{pH} 5.0$ by increasing the supply of the precursors $\beta$-alanine and NADPH. Although both approaches can yield over $10 \mathrm{~g} / \mathrm{L}$ of $3-\mathrm{HP}$ when implemented in yeast, this is still far from the high titers needed for the commercially viable production of commodity chemicals, which typically starts at $100 \mathrm{~g} / \mathrm{L}$ (Kumar et al., 2013). MalonylCoA biosensors and genome-scale engineering tools can be combined to improve the 3-HP production in yeast. Nevertheless, although yeast is an intrinsically acid-tolerant host, which greatly simplifies the downstream process, developing an effective and

\section{REFERENCES}

Bao, Z., HamediRad, M., Xue, P., Xiao, H., Tasan, I., Chao, R., et al. (2018). Genome-scale engineering of Saccharomyces cerevisiae with single nucleotide precision. Nat. Biotechnol. 36, 505-508. doi: 10.1038/nbt.4132

Borodina, I., Kildegaard, K. R., Jensen, N. B., Blicher, T. H., Maury, J., Sherstyk, S., et al. (2015). Establishing a synthetic pathway for high-level production of 3hydroxypropionic acid in Saccharomyces cerevisiae via $\beta$-alanine. Metab. Eng. 27, 57-64. doi: 10.1016/j.ymben.2014.10.003

Bro, C., Regenberg, B., Förster, J., and Nielsen, J. (2006). In silico aided metabolic engineering of Saccharomyces cerevisiae for improved bioethanol production. Metab. Eng. 8, 102-111. doi: 10.1016/j.ymben.2005.09.007

Burk, M. J., and Osterhout, R. E. (2010). Methods and organisms for production of 3-hydroxypropionic acid. U.S. Patent NO 20100021978.

Chen, X., Gao, C., Guo, L., Hu, G., Luo, Q., Liu, J., et al. (2018a). DCEO biotechnology: tools to design, construct, evaluate, and optimize the metabolic pathway for biosynthesis of chemicals. Chem. Rev. 118, 4-72. doi: 10.1021/acs. chemrev.6b00804

Chen, X., Yang, X., Shen, Y., Hou, J., and Bao, X. (2018b). Screening phosphorylation site mutations in yeast acetyl-CoA carboxylase using malonylCoA sensor to improve malonyl-CoA-derived product. Front. Microbiol. 9:47. doi: 10.3389/fmicb.2018.00047

Chen, X., Yang, X., Shen, Y., Hou, J., and Bao, X. (2017). Increasing malonyl$\mathrm{CoA}$ derived product through controlling the transcription regulators of phospholipid synthesis in Saccharomyces cerevisiae. ACS Synth. Biol. 6, 905-912. doi: 10.1021/acssynbio.6b00346

Chen, Y., Bao, J., Kim, I. K., Siewers, V., and Nielsen, J. (2014). Coupled incremental precursor and co-factor supply improves 3-hydroxypropionic acid production in Saccharomyces cerevisiae. Metab. Eng. 22, 104-109. doi: 10.1016/ j.ymben.2014.01.005

Chen, Y., Daviet, L., Schalk, M., Siewers, V., and Nielsen, J. (2013). Establishing a platform cell factory through engineering of yeast acetyl-CoA metabolism. Metab. Eng. 15, 48-54. doi: 10.1016/j.ymben.2012.11.002

Chen, Y., and Nielsen, J. (2016). Biobased organic acids production by metabolically engineered microorganisms. Curr. Opin. Biotechnol. 37, 165-172. doi: 10.1016/j.copbio.2015.11.004 economical 3-HP downstream purification technology could also improve the output of 3-HP after separation and purification (Li et al., 2015; Si et al., 2015, 2017; David et al., 2016; Bao et al., 2018). Further optimization of the recombinant yeast strains and development of more powerful separation techniques could raise the titer and yield of 3-HP enough to enable economically competitive bioproduction at an industrial scale in the future.

\section{AUTHOR CONTRIBUTIONS}

R-YJ, YD, ZG, and X-JJ wrote the manuscript. T-QS, LL, and HH assisted with writing, editing, and finalizing the manuscript.

\section{FUNDING}

This work was financially supported by the National Natural Science Foundation of China (Nos. 21776131 and 21476111), the Program for Innovative Research Teams in Universities of Jiangsu Province, the Priority Academic Program for the Development of Jiangsu Higher Education Institutions, and the Jiangsu Synergetic Innovation Center for Advanced BioManufacture (No. XTD1814).

Chen, Y., Siewers, V., and Nielsen, J. (2012). Profiling of cytosolic and peroxisomal acetyl-CoAmetabolism in Saccharomyces cerevisiae. PLoS One 7:e42475. doi: 10.1371/journal.pone.0042475

Daniel, R., Bobik, T. A., and Gottschalk, G. (1998). Biochemistry of coenzyme B12dependent glycerol and diol dehydratases and organization of the encoding genes. FEMS Microbiol. Rev. 22, 553-566. doi: 10.1111/j.1574-6976.1998. tb00387.x

David, F., Nielsen, J., and Siewers, V. (2016). Flux control at the malonyl-CoA node through hierarchical dynamic pathway regulation in Saccharomyces cerevisiae. ACS Synth. Biol. 5, 224-233. doi: 10.1021/acssynbio.5b00161

Gokarn, R. R., Selifonova, O. V., Jessen, H. J., Gort, S. J., Selmer, T., and Buckel, W. (2011). 3-hydroxypropionic acid and other organic compounds. U.S. Patent No 8076120 .

Guo, Z. P., Zhang, L., Ding, Z. Y., and Shi, G. Y. (2011). Minimization of glycerol synthesis in industrial ethanol yeast without influencing its fermentation performance. Metab. Eng. 13, 49-59. doi: 10.1016/j.ymben.2010.1 1.003

Hoek, P. V., Flikweert, M. T., van der Aart, Q. J. M., Steensma, H. Y., Dijken, J. P. V., and Pronk, J. T. (1998). Effects of pyruvate decarboxylase overproduction on flux distribution at the pyruvate branch point in Saccharomyces cerevisiae. Appl. Environ. Microbiol. 64, 2133-2140.

Holo, H. (1989). Chloroflexus aurantiacus, secretes 3-hydroxypropionate, a possible intermediate in the assimilation of $\mathrm{CO} 2$, and acetate. Arch. Microbiol. 151, 252-256. doi: 10.1007/BF00413138

Hügler, M., Menendez, C., Schägger, H., and Fuchs, G. (2002). Malonylcoenzyme a reductase from chloroflexus aurantiacus, a key enzyme of the 3hydroxypropionate cycle for autotrophic co2 fixation. J. Biol. Chem. 277:12137. doi: 10.1128/JB.184.9.2404-2410.2002

Jessen, H., Rush, B., Huryta, J., Mastel, B., Berry, A., Yaver, D., et al. (2015). Compositions and methods for 3-hydroxypropionic acid production. U.S. Patent No 9090918.

Jiang, X., Meng, X., and Xian, M. (2009). Biosynthetic pathways for 3hydroxypropionic acid production. Appl. Microbiol. Biotechnol. 82, 995-1003. doi: 10.1007/s00253-009-1898-7

Kildegaard, K. R., Hallström, B. M., Blicher, T. H., Sonnenschein, N., Jensen, N. B., Sherstyk, S., et al. (2014). Evolution reveals a glutathione-dependent 
mechanism of 3-hydroxypropionic acid tolerance. Metab. Eng. 26, 57-66. doi: 10.1016/j.ymben.2014.09.004

Kildegaard, K. R., Jensen, N. B., Schneider, K., Czarnotta, E., Özdemir, E., Klein, T., et al. (2016). Engineering and systems-level analysis of Saccharomyces cerevisiae for production of 3-hydroxypropionic acid via malonyl-CoA reductasedependent pathway. Microb. Cell Fact. 15:53. doi: 10.1186/s12934-016-0 451-5

Kildegaard, K. R., Wang, Z., Chen, Y., Nielsen, J., and Borodina, I. (2015). Production of 3-hydroxypropionic acid from glucose and xylose by metabolically engineered Saccharomyces cerevisiae. Metab. Eng. Commun. 2, 132-136. doi: 10.1016/j.ymben.2016.11.009

Ko, Y., Ashok, S., Ainala, S. K., Sankaranarayanan, M., Chun, A. Y., Jung, G. Y., et al. (2014). Coenzyme B12 can be produced by engineered Escherichia coli under both anaerobic and aerobic conditions. Biotechnol. J. 9, 1526-1535. doi: 10.1002/biot.201400221

Kocharin, K., Chen, Y., Siewers, V., and Nielsen, J. (2012). Engineering of acetylCoA metabolism for the improved production of polyhydroxybutyrate in Saccharomyces cerevisiae. AMB Express 2:52. doi: 10.1186/2191-0855-2-52

Kumar, V., Ashok, S., and Park, S. (2013). Recent advances in biological production of 3-hydroxypropionic acid. Biotechnol. Adv. 31, 945-961. doi: 10.1016/j. biotechadv.2013.02.008

Lan, E. I., Chuang, D. S., Shen, C. R., Lee, A. M., Ro, S. Y., and Liao, J. C. (2015). Metabolic engineering of Cyanobacteria for photosynthetic 3hydroxypropionic acid production from CO2 using Synechococcu PCC 7942. Metab. Eng. 31, 163-170. doi: 10.1016/j.ymben.2015.08.002

Li, S., Si, T., Wang, M., and Zhao, H. (2015). Development of a synthetic malonylCoA sensor in Saccharomyces cerevisiae for intracellular metabolite monitoring and genetic screening. ACS Synth. Biol. 4, 1308-1315. doi: 10.1021/acssynbio. 5 b00069

Lian, J., Mishra, S., and Zhao, H. (2018). Recent advances in metabolic engineering of saccharomyces cerevisiae: new tools and their applications. Metab. Eng. doi: 10.1016/j.ymben.2018.04.011 [Epub ahead of print].

Lian, J., Si, T., Nair, N. U., and Zhao, H. (2014). Design and construction of acetylCoA overproducing Saccharomyces cerevisiae strains. Metab. Eng. 24, 139-149. doi: 10.1016/j.ymben.2014.05.010

Liao, H. H., Gokarn, R. R., Gort, S. J., Jessen, H. J., and Selifonova, O. (2010). Alanine 2,3-Aminomutase. U.S. Patent NO 7309597, B2.

Liao, H. H., Gokarn, R. R., Gort, S. J., Jessen, H. J., and Selifonova, O. V. (2007). Production of 3-hydropropionic acid using beta-alanine/pyruvate aminotransferase. U.S. Patent NO 20070107080, A1. doi: 10.1016/j.ymben.2014. 10.003

Liu, C., Ding, Y., Xian, M., Liu, M., Liu, H., Ma, Q., et al. (2017). Malonyl-CoA pathway: a promising route for 3-hydroxypropionate biosynthesis. Crit. Rev. Biotechnol. 37, 933-941. doi: 10.1080/07388551.2016.1272093

Liu, C., Ding, Y., Zhang, R., Liu, H., Xian, M., and Zhao, G. (2016). Functional balance between enzymes in malonyl-CoA pathway for 3-hydroxypropionate biosynthesis. Metab. Eng. 34, 104-111. doi: 10.1016/j.ymben.2016.01.001

Liu, C., Wang, Q., Xian, M., Ding, Y., and Zhao, G. (2013). Dissection of malonylcoenzyme A reductase of Chloroflexus aurantiacus results in enzyme activity improvement. PLoS One 8:e75554. doi: 10.1371/journal.pone.0075554

Lynch, M. D., Gill, R. T., and Lipscomb, T. E. W. (2016). Method for producing 3-hydroxypropionic acid and other products. U.S. Patent NO 9428778.

Maury, J., Kannan, S., Jensen, N. B., Öberg, F. K., Kildegaard, K. R., Forster, J., et al. (2018). Glucose-dependent promoters for dynamic regulation of metabolic pathways. Front. Bioeng. Biotechnol. 6:63. doi: 10.3389/fbioe.2018.00063

Nielsen, J. (2014). Synthetic biology for engineering acetyl coenzyme a metabolism in yeast. $m$ Bio 5:e02153. doi: $10.1128 / \mathrm{mBio} .02153-14$

Nielsen, J., and Keasling, J. D. (2016). Engineering cellular metabolism. Cell 164, 1185-1197. doi: 10.1016/j.cell.2016.02.004

Pina, C. D., Falletta, E., and Rossi, M. (2011). A green approach to chemical building blocks: the case of 3-hydroxypropanoic acid. Green Chem. 13, 1624-1632. doi: 10.1039/c1gc15052a
Pronk, J. T., Yde, S. H., and van Dijken, J. P. (1996). Pyruvate metabolism in Saccharomyces cerevisiae. Yeast 12, 1607-1633. doi: 10.1002/(SICI) 10 97-0061(199612)12:16<1607::AID-YEA70>3.0.CO;2-4

Rathnasingh, C., Raj, S. M., Lee, Y., Catherine, C., Ashok, S., and Park, S. (2012). Production of 3-hydroxypropionic acid via malonyl-CoA pathway using recombinant Escherichia coli strains. J. Biotechnol. 157, 633-640. doi: 10.1016/j. jbiotec.2011.06.008

Roth, J. R., Lawrence, J. G., and Bobik, T. A. (1996). Cobalamin (coenzyme B12): synthesis and biological significance. Ann. Rev. Microbiol. 50, 137-181. doi: 10.1146/annurev.micro.50.1.137

Shi, S., Chen, Y., Siewers, V., and Nielsen, J. (2014). Improving production of malonyl coenzyme A-derived metabolites by abolishing snf1-dependent regulation of acc. mBio 5:e01130-14. doi: 10.1128/mBio.01130- 14

Si, T., Chao, R., Min, Y., Wu, Y., Ren, W., and Zhao, H. (2017). Automated multiplex genome-scale engineering in yeast. Nat. Commun. 8:15187. doi: $10.1038 /$ ncomms 15187

Si, T., Luo, Y., Bao, Z., and Zhao, H. (2015). Rnai-assisted genome evolution in saccharomyces cerevisiae for complex phenotype engineering. ACS Synth. Biol. 4, 283-291. doi: 10.1021/sb500074a

Strauss, G., Eisenreich, W., Bacher, A., and Fuchs, G. (1992). 13C-NMR study of autotrophic $\mathrm{CO} 2$ fixation pathways in the sulfur-reducing archaebacterium Thermoproteus neutrophilus and in the phototrophic eubacterium Chloroflexus aurantiacus. FEBS J. 205, 853-866. doi: 10.1111/j.1432-1033.1992.tb16850.x

Tang, X., Lee, J., and Chen, W. N. (2015). Engineering the fatty acid metabolic pathway in Saccharomyces cerevisiae, for advanced biofuel production. Metab. Eng. Commun. 2, 58-66. doi: 10.1016/j.meteno.2015.06.005

Tehlivets, O., Scheuringer, K., and Kohlwein, S. D. (2007). Fatty acid synthesis and elongation in yeast. Biochim. Biophys. Acta 1771, 255-270. doi: 10.1016/j.bbalip. 2006.07.004

Toraya, T. (2002). Enzymatic radical catalysis: coenzyme B12-dependent diol dehydratase. Chem. Rec. 2, 352-366. doi: 10.1002/tcr.10035

Valdehuesa, K. N., Liu, H., Nisola, G. M., Chung, W. J., Lee, S. H., and Si, J. P. (2013). Recent advances in the metabolic engineering of microorganisms for the production of 3-hydroxypropionic acid as C3 platform chemical. Appl. Microbiol. Biotechnol. 97, 3309-3321. doi: 10.1007/s00253-013-4802-4

Verho, R., Londesborough, J., Penttilä, M., and Richard, P. (2003). Engineering redox cofactor regeneration for improved pentose fermentation in Saccharomyces cerevisiae. Appl. Environ. Microbiol. 69, 5892-5897. doi: 10.1128/AEM.69.10.5892-5897.2003

Werpy, T., Petersen, G., Aden, A., Bozell, J., Holladay, J., White, J., et al. (2004). Top Value Added Chemicals from Biomass. Volume 1-Results of Screening for Potential Candidates from Sugars and Synthesis Gas. Available at: https://www. energy.gov/sites/prod/files/2014/03/f14/35523.pdf

Woods, A., Munday, M. R., Scott, J., Yang, X., Carlson, M., and Carling, D. (1994). Yeast snf1 is functionally related to mammalian amp-activated protein kinase and regulates acetyl-CoA carboxylase in vivo. J. Biol. Chem. 269, 19509-19515.

Zhang, D., Hillmyer, M. A., and Tolman, W. B. (2004). A new synthetic route to poly[3-hydroxypropionic acid] (p[3-HP]): ring-opening polymerization of 3HP macrocyclic esters. Macromolecules 37, 8198-8200. doi: 10.1021/ma048092q

Conflict of Interest Statement: The authors declare that the research was conducted in the absence of any commercial or financial relationships that could be construed as a potential conflict of interest.

Copyright () 2018 Ji, Ding, Shi, Lin, Huang, Gao and Ji. This is an open-access article distributed under the terms of the Creative Commons Attribution License (CC BY). The use, distribution or reproduction in other forums is permitted, provided the original author(s) and the copyright owner(s) are credited and that the original publication in this journal is cited, in accordance with accepted academic practice. No use, distribution or reproduction is permitted which does not comply with these terms. 